\title{
Clinical and paraclinical manifestations in patients suspected of being infected with COVID-19
}

\author{
${ }^{\star}$ Ion Sirbu, Sergiu Matcovschi \\ Discipline of Clinical Synthesis, Department of Internal Medicine \\ Nicolae Testemitanu State University of Medicine and Pharmacy, Chisinau, the Republic of Moldova \\ Authors' ORCID iDs, academic degrees and contributions are available at the end of the article \\ ${ }^{\star}$ Corresponding author - Ion Sirbu, e-mail: ion.sirbu@usmf.md \\ Manuscript received August 04, 2021; revised manuscript October 01, 2021; published online October 12, 2021
}

\begin{abstract}
Background: It was intended to study the structure of the symptoms in patients suspected of having SARS-CoV-2 virus infection, as well as to find any correlations between the clinical, paraclinical and radiological manifestations in positive versus negative patients, in order to further facilitate the diagnosis and triage of patients.

Material and methods: 101 patients seeking medical attendance at the COVID-19 Triage Center in Chisinau have been examined, presenting various respiratory symptoms. The frequency of symptoms and the results of the paraclinical investigations were evaluated based on the results of the PCR tests for SARS-CoV-2 infection and the assessment of correlations (Pearson).

Results: Out of 101 subjects, 50 tested SARS-CoV-2 positive, and the remaining 51 - negative. The clinical manifestations of SARS-CoV-2 suspects were as follows: fatigue $-72 \%$, sweating $-54 \%$, chills $-52 \%$, fever $-49 \%$, subfebrility $-39 \%$, myalgias and arthralgias $-37 \%$, cough $-35 \%$ (sputum $-17 \%$ and hemoptysis $-2 \%$ ), dyspnea $-34 \%$, chest pain $-23 \%$, anosmia $-12 \%$, headache $-11 \%$, dyspeptic syndrome $-8 \%$. Infiltrates on chest radiography were found in $22 \%$ of cases.

A weak inverse correlation $(R=-0.22, P<0.05)$ between the leukocyte count and SARS-CoV-2 test results was found. An average direct correlation between the presence of fever $(\mathrm{R}=0.36, \mathrm{P}<0.05)$ and a positive COVID-19 test was also noticed.

Conclusions: Certain symptoms such as anosmia were more commonly seen in patients with positive COVID-19 tests. The absence of pulmonary infiltrates and the presence of dyspnoea have been negative predictive factors for COVID-19. Leukopenia has been noticed only in SARS-CoV-2 positive patients. Subfebrility has not shown a predictive significance of COVID-19.

Key words: COVID-19, dyspnea, leukopenia, anosmia.
\end{abstract}

Cite this article

Sirbu I, Matcovschi S. Clinical and paraclinical manifestations in patients suspected of being infected with COVID-19. Mold Med J. 2021;64(4):62-65. https://doi.org/10.52418/moldovan-med-j.64-4.21.11.

\section{Introduction}

COVID-19 is a respiratory condition where pulmonary manifestations are the main clinical presentations of the disease. According to the reported studies, SARS-CoV-2 infection is not limited to the respiratory system, it does affect other organs, as well. Renal dysfunction, gastrointestinal complications, liver failures, cardiac manifestations, neurological abnormalities and hematological manifestations are among the reported extrapulmonary features [1].

Studies report a timeframe of 6 to 41 days from the onset of COVID-19 symptoms until death, with a median of 14 days [2].

The most commonly reported symptoms at the onset of COVID-19 disease are fever, cough, and fatigue, while other symptoms include the presence of sputum, headache, hemoptysis, diarrhea, dyspnea, and lymphopenia [3-5].

Laboratory tests showed leukopenia, of which $70 \%$ were neutrophils. Additionaly, increased values of blood $\mathrm{C}$-reactive protein were observed. A high sedimentation rate of erythrocytes and elevated D-dimers have also been found [6].

Studies report the presence of various common symptoms for many viral respiratory diseases such as: fever $-83 \%$, cough $-82 \%$, dyspnea $-31 \%$, myalgia $-11 \%$, confusion $9 \%$, headache $-8 \%$, sore throat $-5 \%$, rhinorrhea $-4 \%$, chest pain $-2 \%$, diarrhea $-2 \%$, nausea and vomiting $-1 \%$ [7]

Therefore, the early diagnosis of SARS-CoV-2 infection has become a challenge for the clinician, and the delayed results of the PCR tests, as well as the presence of false-negative results, have made it even more difficult to establish the diagnosis. Currently there is no test with a specificity and sensitivity of $100 \%$ [8].

Thus, it was intended to study the structure of symptoms in patients suspected of having the SARS-CoV-2 virus infection, and to find the correlations between the clinical and paraclinical manifestations in positive versus negative patients, in order to further facilitate the diagnosis and triage of patients. 


\section{Material and methods}

The current study involved 101 patients (51 men and 50 women), suspected of having the SARS-CoV-2 infection, who sought medical attendance at the Covid-19 Center in Chisinau between April and July 2020, in a state of mild and average severity, presenting various respiratory symptoms. The patients' age varied between 18 and 84 (the average age being 49.3 years). The disease history was collected and a paraclinical examination was performed (full blood count, chest radiography, ECG, SARS-CoV-2 PCR test). The frequency of symptoms was assessed according to the results of the PCR tests for SARS-CoV-2 and the assessment of the correlations (Pearson) between clinical and paraclinical data.

The statistical data was processed using the SPSS program, with a value of „p” less than 0.05 being considered statistically significant.

\section{Results}

51 negative and 50 positive tests for SARS-CoV-2 were obtained. The clinical manifestations (in descending order) of SARS-CoV-2 positive patients were as follows: fatigue $72 \%$, sweating $-54 \%$, chills $-52 \%$, fever $-49 \%$, subfebrility - 39\%, myalgias and arthralgias - 37\%, cough - 35\% (sputum $-17 \%$ and hemoptysis $-2 \%$ ), dyspnea $-34 \%$, chest pain - $23 \%$, anosmia - $12 \%$, headache $-11 \%$, dyspeptic syndrome $-8 \%$ (fig. 1). The presence of infiltrates on chest radiography was identified by performing a chest radiography in 21 patients, of which 12 patients were COVID-19 positive and 9 had negative SARS-CoV-2 tests.

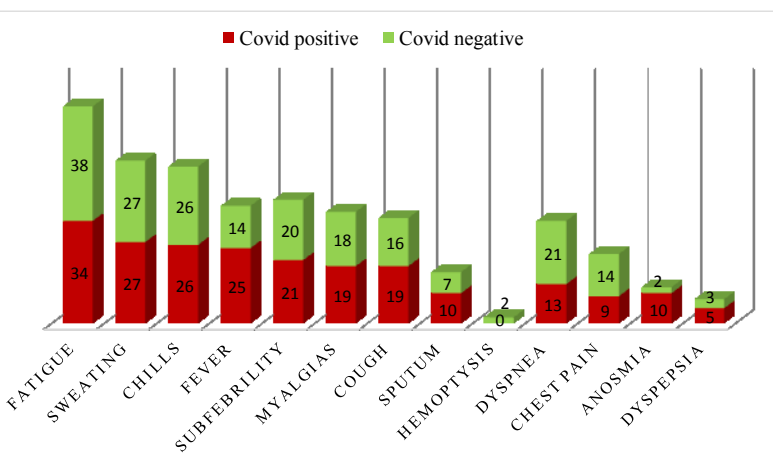

Fig. 1. Structure of symptoms according to the results of the SARS-CoV-2 test

The number of days from the onset of symptoms until testing varied from 1 to 60 days (average length 7.08 days), the results being different depending on the test result. Thus, it was observed that the earlier the onset of symptoms, the greater the chances of testing positive for COVID-19 (fig. 2).

A great number of the suspects presented with multiple comorbidities, making it more difficult to establish the diagnosis, many symptoms being similar for other pathologies (fig. 3).

In $55 \%$ of the patients with a negative test, at least one concomitant pathology has been detected. And in those with positive results, multiple morbidity was detected in $58 \%$ of cases.

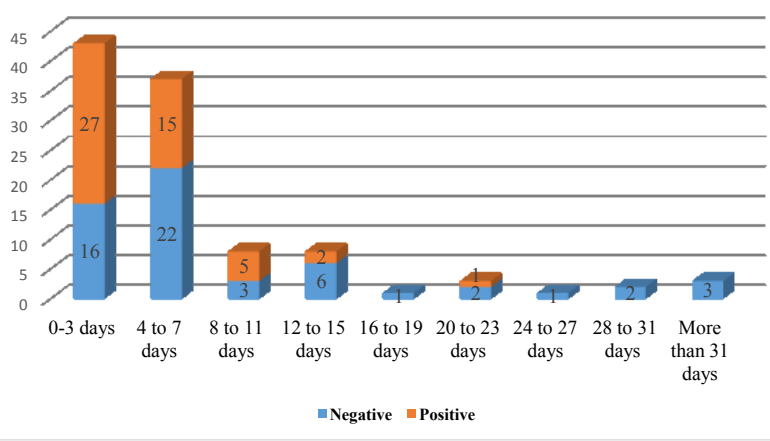

Fig. 2. The number of days from the disease onset

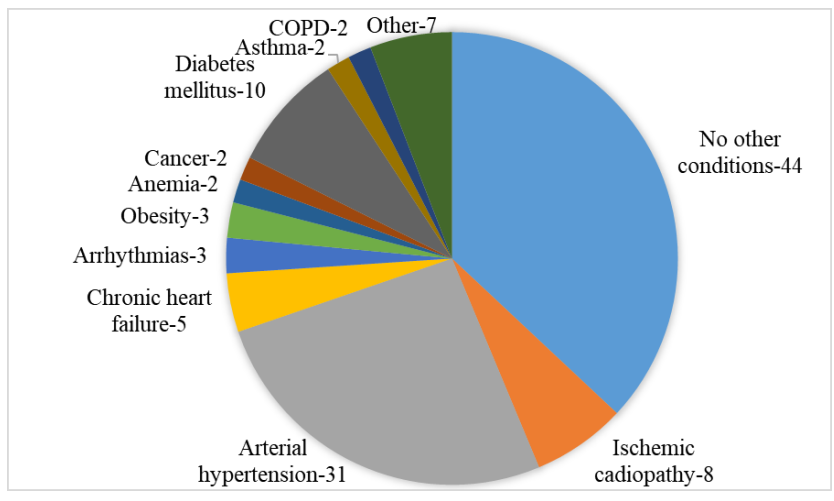

Fig. 3. Structure of multiple morbidities in COVID-19 suspects

The $\mathrm{O}_{2}$ saturation of the patients was measured on admission, thus values between $83 \%$ and $100 \%$ were obtained (the average $\mathrm{SaO}_{2}$ being 96.83\%). A weak correlation was measured between the saturation on admision and the SARS-CoV-2 test result, being statistically insignificant $(\mathrm{R}=$ $0.06 ; \mathrm{p}>0.05)$.

A full blood count was performed in all patients, leukocyte values and erythrocyte sedimentation rate (ESR) were also investigated. A weak, statistically significant inverse correlation was found between the leukocyte numbers and the SARS-CoV-2 test results ( $\mathrm{R}=-0.222 ; \mathrm{p}<0.05)$, (fig. 4).

Lymphocyte values were also assessed compared to the COVID-19 test results. No correlation was determined between the lymphocyte count and SARS-CoV-2 test results (fig. 5).

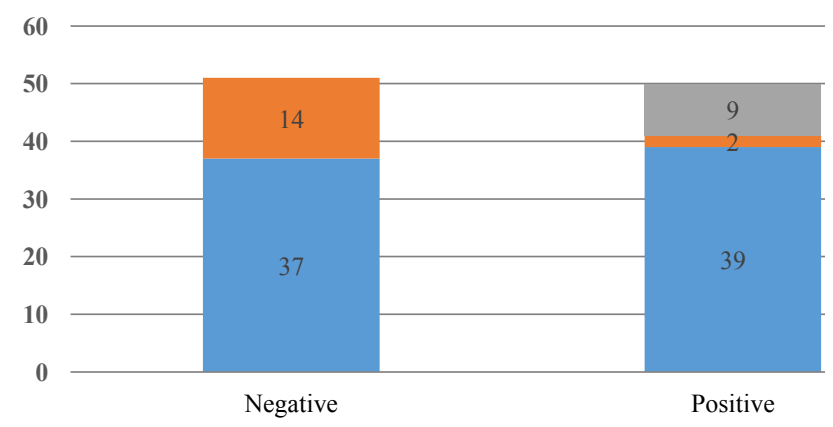

Fig. 4. COVID-19 test results and leukocyte values 


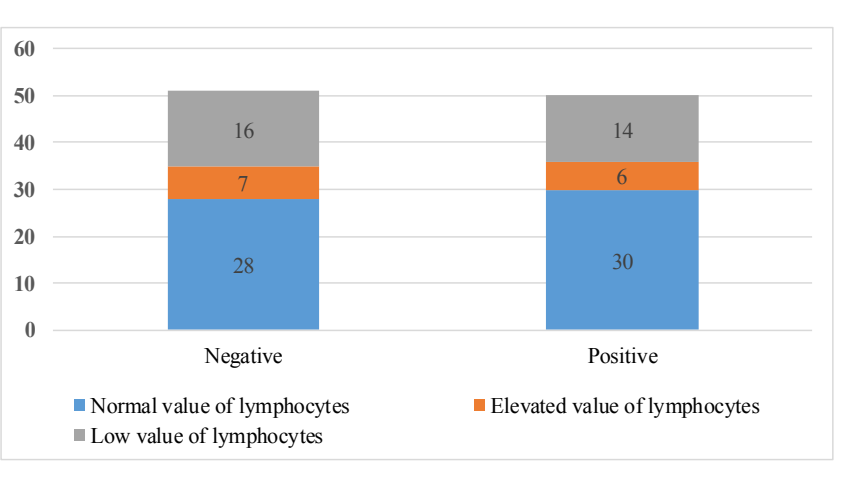

Fig. 5. COVID-19 test results and lymphocyte values

The correlation between the SARS-CoV-2 test results and the ESR values was also measured, obtaining a weak direct correlation, being statistically insignificant $(\mathrm{R}=0.04$; $\mathrm{p}>0.05)$.

A mean direct correlation $(\mathrm{R}=0.41, \mathrm{P}<0.05)$ was found between dyspnea and the presence of pulmonary infiltrates on chest radiography in SARS-CoV-2 positive patients and its absence in patients with negative test results $(R=0.18$, $\mathrm{P}>0.1$.

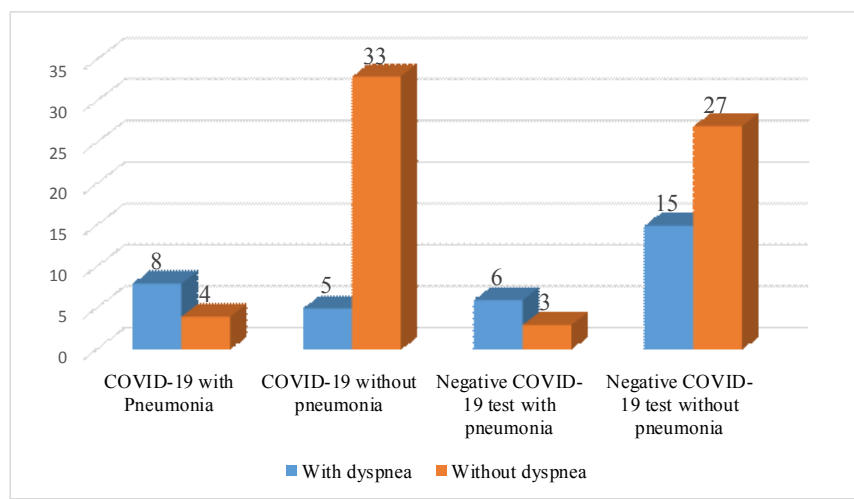

Fig. 6. Correlation between dyspnea and pulmonary infiltrates based on the test results

An average direct correlation was noticed between fe$\operatorname{ver}(\mathrm{R}=0.31, \mathrm{P}<0.05)$ and pneumonia in the SARS-CoV-2 positive patients, with its absence in subjects with negative test results. An average direct correlation between fever $(\mathrm{R}=0.36, \mathrm{P}<0.05)$ and a positive COVID-19 test result was also observed. Subfebrility was found to be of approximately the same frequency in patients with positive and negative tests for COVID-19.

\section{Discussion}

Within the current observational study based on data gained from 101 patients suspected of having a COVID-19 infection, the presence of many symptoms common for respiratory tract infections was noticed, the most common of them being fever, signs of general intoxication and cough. Also, some symptoms such as anosmia have been detected much more frequently in patients with positive SARS-CoV-2 test results. Other studies in the field have shown similar results [9].

Furthermore, dyspnea and chest pain were more common in patients with negative SARS-CoV-2 test results. This phenomenon could be explained by the presence of multiple concomitant pathologies that were also suspected, such as ischemic heart disease, cardiac failure or pulmonary obstructive pathologies [10].

Low leukocyte values in suspect patients could be a strong predictor of SARS-CoV-2 infection, as all 9 patients with leukopenia were diagnosed with COVID-19. Leukocytosis has been found more frequently in patients with negative test results, which could be explained by exacerbation of chronic conditions, such as asthma, chronic obstructive pulmonary disease or some acute conditions such as pyelonephritis.

The values of lymphocytes in suspect patients was of no significance in establishing the presumptive diagnosis of COVID-19, as lymphopenia and lymphocytosis were also present in patients with negative test results. These results refer to patients with a mild and average severity of COVID-19 [11].

Dyspnea was more common in patients with negative SARS-CoV-2 tests, being present in patients who also had radiological imaging findings, but the presence of dyspnea without radiological changes was also a negative predictor of COVID-19. Fever and subfebrility were found in $80 \%$ of patients.

\section{Conclusions}

1. Anosmia was found 5 times more frequently in testpositive patients, thus being a strong predictor in a suspect case of COVID-19. Other symptoms were found with approximately the same frequency in patients with negative COVID-19 test results.

2. Dyspnea without any lung damage is 3.6 times more common in patients with negative COVID-19 test results.

3. Lung damage was 1.3 times more common in patients with positive versus negative test results.

4. $33 \%$ of patients with COVID-19 and pneumonia had no dyspnea.

5. Leukopenia was found in $9 \%$ of suspects and only in patients with positive SARS-CoV-2 test results.

6. Fever was 1.8 times more common in patients with positive COVID-19 test results; however, subfebrility was found in a ratio of 1:1 within the study group.

\section{References}

1. Behzad S, Aghaghazvini L, Radmard AR, Gholamrezanezhad A. Extrapulmonary manifestations of COVID-19: Radiologic and clinical overview. Clin Imaging. 2020 Oct;66:35-41. doi: 10.1016/j. clinimag.2020.05.013.

2. Wang W, Tang J, Wei F. Updated understanding of the outbreak of 2019 novel coronavirus (2019-nCoV) in Wuhan, China. J Med Virol 2020;92(4):441-447. doi: 10.1002/jmv.25689.

3. Ren LL, Wang YM, Wu ZQ, Xiang ZC, Guo L, Xu T. Identification of a novel coronavirus causing severe pneumonia in human: a descriptive study. Chinese Med J. 2020;133(9):1015-1024. doi: 10.1097/ CM9.0000000000000722. 
4. Huang C, Wang Y, Li X, Ren L, Zhao J, Hu Y. Clinical features of patients infected with 2019 novel coronavirus in Wuhan, China. Lancet. 2020;395(10223):497-506. doi: 10.1016/S0140-6736(20)30183-5.

5. Carlos WG, Dela Cruz CS, Cao B, Pasnick S, Jamil S. Novel wuhan (2019-nCoV) coronavirus. Am J Respir Crit Care Med. 2020;201(4):7-8. doi: $10.1164 / \mathrm{rccm} .2014$ P7.

6. Lei J, Li J, Li X, Qi X. CT imaging of the 2019 novel coronavirus (2019-nCoV) pneumonia. Radiology. 2020;295(1):18. doi: 10.1148/ radiol.2020200236.

7. Chen N, Zhou M, Dong X, Qu J, Gong F, Han Y, et al. Epidemiological and clinical characteristics of 99 cases of 2019 novel coronavirus pneumonia in Wuhan, China: a descriptive study. Lancet. 2020;395(10223):507-513. doi: 10.1016/S0140-6736(20)30211-7.
8. Kost GJ. The impact of increasing disease prevalence, false omissions, and diagnostic uncertainty on coronavirus disease 2019 (COVID-19) test performance. Arch Pathol Lab Med. 2021;145(7):797-813. doi: 10.5858/ arpa.2020-0716-SA.

9. Yang X, Yu Y, Xu J, et al. Clinical course and outcomes of critically ill patients with SARS-CoV-2 pneumonia in Wuhan, China: a singlecentered, retrospective, observational study. Lancet Resp Med. 2020 May;8(5):475-481. doi: 10.1016/S2213-2600(20)30079-5.

10. Ejaz H, Alsrhani A, Zafar A, Javed H, Junaid K, Abdalla AE, et al. COVID-19 and comorbidities: deleterious impact on infected patients. J Infect Public Health. 2020;13(12):1833-1839. doi: 10.1016/j.jiph.2020. 07.014 .

11. Gustine JN, Jones D. Immunopathology of Hyperinflammation in COVID-19. Am J Pathol. 2021;191(1):4-17. doi: 10.1016/j.ajpath.2020. 08.009.

\section{Authors' ORCID iDs and academic degrees}

Ion Sirbu, MD, PhD Applicant - https://orcid.org/0000-0003-1072-4371

Sergiu Matcovschi, MD, PhD, Professor of Pneumology - https://orcid.org/0000-0003-1623-930X

\section{Authors' contributions}

IS conceptualized the project and drafted the first manuscript. SM interpreted the data and critically revised the manuscript. Both authors revised and critically approved the final version of the manuscript.

\section{Funding}

This study was supported by Nicolae Testemitanu State University of Medicine and Pharmacy. The trial was the authors' initiative. The authors are independent and take responsibility for the integrity of the data and accuracy of the data analysis.

Ethics approval and consent to participate

The study was approved by the Research Ethics Committee of Nicolae Testemitanu State University of Medicine and Pharmacy, proceedings No 04 of 16.02.2021.

\section{Conflict of Interests}

No competing interests were disclosed. 